\title{
The Characterization of Generalized Jordan Centralizers on Triangular Algebras
}

\author{
Quanyuan Chen ${ }^{\mathbb{D}},{ }^{1}$ Xiaochun Fang $\mathbb{D}^{2},{ }^{2}$ and Changjing $\mathrm{Li}^{3}$ \\ ${ }^{1}$ College of Infor., Jingdezhen Ceramic Institute, Jingdezhen, Jiangxi 333403, China \\ ${ }^{2}$ Department of Mathematics, Tongji University, Shanghai 200092, China \\ ${ }^{3}$ Department of Mathematics, Shandong Normal University, Jinan, Shandong 250014, China
}

Correspondence should be addressed to Quanyuan Chen; cqy0798@163.com

Received 6 May 2018; Revised 27 August 2018; Accepted 30 August 2018; Published 16 September 2018

Academic Editor: Ismat Beg

Copyright (C) 2018 Quanyuan Chen et al. This is an open access article distributed under the Creative Commons Attribution License, which permits unrestricted use, distribution, and reproduction in any medium, provided the original work is properly cited.

In this paper, it is shown that if $\mathscr{T}=\operatorname{Tr} i(\mathscr{A}, \mathscr{M}, \mathscr{B})$ is a triangular algebra and $\phi$ is an additive operator on $\mathscr{T}$ such that $(m+n+$ $k+l) \phi\left(T^{2}\right)-\left(m \phi(T) T+n T \phi(T)+k \phi(I) T^{2}+l T^{2} \phi(I)\right) \in \mathbb{F} I$ for any $T \in \mathcal{T}$, then $\phi$ is a centralizer. It follows that an $(m, n)-$ Jordan centralizer on a triangular algebra is a centralizer.

\section{Introduction and Preliminaries}

The triangular algebras are firstly introduced in [1]. Let $\mathscr{A}$ and $\mathscr{B}$ be two unital algebras over a commutative ring $\mathscr{R}$, where $\mathscr{R}$ is a unital commutative ring, and let $\mathscr{M}$ be a unital $(\mathscr{A}, \mathscr{B})-$ bimodule. Suppose that $\mathscr{M}$ is a faithful $(\mathscr{A}, \mathscr{B})$ - bimodule; that is, $\mathscr{M}$ is an $(\mathscr{A}, \mathscr{B})$ - bimodule satisfying $A \in \mathscr{A}: A \mathscr{M}=0$ and implies that $A=0$, and $B \in \mathscr{B}: \mathscr{M B}=0$ implies that $B=0$. Consider the set

$$
\begin{aligned}
\mathscr{T} & =\operatorname{Tri}(\mathscr{A}, \mathscr{M}, \mathscr{B}) \\
& =\left\{\left(\begin{array}{rr}
A & M \\
B
\end{array}\right): A \in \mathscr{A}, M \in \mathscr{M}, B \in \mathscr{B}\right\} .
\end{aligned}
$$

The usual matrix addition and matrix multiplication on $\operatorname{Tr}(\mathscr{A}, \mathscr{M}, \mathscr{B})$ are defined as follows: for any $A_{1}, A_{2} \in$ $\mathscr{A}, M_{1}, M_{2} \in \mathscr{M}, B_{1}, B_{2} \in \mathscr{B}$,

$$
\begin{aligned}
& \left(\begin{array}{cc}
A_{1} & M_{1} \\
& B_{1}
\end{array}\right)+\left(\begin{array}{cc}
A_{2} & M_{2} \\
& B_{2}
\end{array}\right)=\left(\begin{array}{cc}
A_{1}+A_{2} & M_{1}+M_{2} \\
& B_{1}+B_{2}
\end{array}\right), \\
& \left(\begin{array}{cc}
A_{1} & M_{1} \\
& B_{1}
\end{array}\right)\left(\begin{array}{cc}
A_{2} & M_{2} \\
& B_{2}
\end{array}\right)=\left(\begin{array}{cc}
A_{1} A_{2} & A_{1} M_{2}+M_{1} B_{2} \\
& B_{1} B_{2}
\end{array}\right) .
\end{aligned}
$$

It is easy to see that $\mathscr{T}=\operatorname{Tr} i(\mathscr{A}, \mathscr{M}, \mathscr{B})$ is an algebra under the usual matrix addition and matrix multiplication and is called a triangular algebra. We denote $\left(\mathscr{A} \mathscr{M}_{\mathscr{B}}\right)$ by $\operatorname{Tri}(\mathscr{A}, \mathscr{M}, \mathscr{B})$ for simplicity.

Let $\mathscr{R}$ be a ring or an algebra and $\phi$ be an additive mapping on $\mathscr{R}$. If $\phi(A B)=\phi(A) B$ (resp., $\phi(A B)=A \phi(B))$ for any $A, B \in \mathscr{R}$, then $\phi$ is called a left centralizer (resp., a right centralizer). A centralizer of $\mathscr{R}$ is an additive mapping which is both a left centralizer and a right centralizer. An additive mapping $\phi: \mathscr{R} \longrightarrow \mathscr{R}$ is called a left (resp., right) Jordan centralizer, if $\phi\left(A^{2}\right)=\phi(A) A$ (resp., $\left.\phi\left(A^{2}\right)=A \phi(A)\right)$ for any $A \in \mathscr{R}$. A Jordan centralizer of $\mathscr{R}$ is an additive mapping which is both a left Jordan and a right Jordan centralizer. An $(m, n)$ - Jordan centralizer is defined in ([2]) as follows: let $\mathscr{R}$ be a ring or an algebra and $\phi$ be an additive mapping on $\mathscr{R}$. If there are $m, n \in \mathbb{N}: m+n \neq 0$ such that $(m+n) \phi\left(A^{2}\right)=$ $m \phi(A) A+n A \phi(A)$ for any $A \in \mathscr{R}$, then $\phi$ is called an $(m, n)-$ Jordan centralizer.

The characterization of centralizers on algebras or rings is a subject in various areas. Bresar and Zalar ([3]) proved that if $\mathscr{R}$ is a prime ring and $\phi$ is an additive mapping on $\mathscr{R}$ such that $\phi\left(A^{2}\right)=\phi(A) A$ (resp., $\left.\phi\left(A^{2}\right)=A \phi(A)\right)$ for any $A \in \mathscr{R}$, then $\phi$ is a left (resp., a right) centralizer. Zalar ([4]) generalized the result to 2 -torsion free semiprime rings as follows: if $\mathscr{R}$ is a 2 -torsion free semiprime ring and $\phi$ is an additive mapping on $\mathscr{R}$ such that $\phi\left(A^{2}\right)=\phi(A) A$ (resp., $\left.\phi\left(A^{2}\right)=A \phi(A)\right)$ for any $A \in \mathscr{R}$, then $\phi$ is a left (resp., a right) centralizer. Vukman ([5]) proved that if $\mathscr{R}$ is a 2 -torsion 
free semiprime ring and $\phi$ is an additive mapping on $\mathscr{R}$ such that $2 \phi\left(A^{2}\right)=\phi(A) A+A \phi(A)$ for any $A \in \mathscr{R}$, then $\phi$ is a centralizer. Benkovic and Eremita ([6]) proved that if $\mathscr{R}$ is a prime ring with $C h(\mathscr{R})=0$ or $C h(\mathscr{R}) \geq n$, where $n$ is a fixed positive integer such that $n \geq 2$, and $\phi$ is an additive mapping on $\mathscr{R}$ such that $\phi\left(A^{n}\right)=\phi(A) A^{n-1}$ for any $A \in \mathscr{R}$, then $\phi$ is a centralizer. Vukman and Kosi-Ulbl ([7]) proved that if $X$ is a Banach space over the field $\mathbb{F}$, and $\mathscr{A}$ is a standard subalgebra of $B(X)$ and $\phi: \mathscr{A} \longrightarrow B(X)$ is an additive mapping such that $\phi\left(A^{m+n+1}\right)=A^{m} \phi(A) A^{n}$ for any $A \in \mathscr{A}$, where $m, n \in \mathbb{Z}^{+}$, then $\phi$ is a centralizer. Qi et al. ([8]) proved that if $\mathscr{A}$ is a standard subalgebra of $B(X)$ with the identity $I$ and $\phi: \mathscr{A} \longrightarrow B(X)$ is an additive mapping such that $\phi\left(A^{m+n+1}\right)-A^{m} \phi(A) A^{n} \in \mathbb{F} I$ for any $A \in \mathscr{A}$, where $X$ is a Banach space over the field $\mathbb{F}$ and $m, n \in \mathbb{Z}^{+}$, then $\phi$ is a centralizer. Yang and Zhang ([9]) proved that, if $\phi: \tau(\mathcal{N}) \longrightarrow \tau(\mathscr{N})$ is an additive mapping on a nest algebra $\tau(\mathcal{N})$, such that $(m+n) \phi\left(A^{p+1}\right)=m \phi(A) A^{p}+n A^{p} \phi(A)$ or $\phi\left(A^{m+n+1}\right)=A^{m} \phi(A) A^{n}$ for any $A \in \tau(\mathcal{N})$, where $\mathcal{N}$ is a nontrivial nest on $H, \tau(\mathscr{N})$ is the corresponding nest algebra, and $m, n, p \in \mathbb{Z}^{+}$, then $\phi$ is a centralizer. J. Vukman ([2]) proved that an $(m, n)$ - Jordan centralizer on a prime ring with $C h(\mathscr{R}) \neq 6 m n(m+n)$ is a centralizer. Li et al. ([10]) proved that a Jordan centralizer on a CSL subalgebra of a von Neumann algebra is a centralizer. Chen et al. ([11]) proved that an additive mapping $\phi$ on a CSL subalgebra of a von Neumann algebra such that $(m+n+k+l) \phi\left(A^{2}\right)-(m \phi(A) A+$ $\left.n A \phi(A)+k \phi(I) A^{2}+l A^{2} \phi(I)\right) \in \mathbb{F} I$ for any $A \in \mathscr{A}$ is a centralizer. Lately several authors investigated $(m, n)-$ Jordan centralizers or their related mappings on rings and algebras. Some of the results can be found in ([5, 12-20]).

Motivated by these results, we are concerned with an additive mapping $\phi$ such that $(m+n+k+l) \phi\left(A^{2}\right)-(m \phi(A) A+$ $\left.n A \phi(A)+k \phi(I) A^{2}+l A^{2} \phi(I)\right) \in \mathbb{F} I$ for any $A \in \mathscr{A}$. It is shown that if $\mathscr{T}=\operatorname{Tr} i(\mathscr{A}, \mathscr{M}, \mathscr{B})$ is a triangular algebra and $\phi$ is an additive operator on $\mathscr{T}$ such that $(m+n+k+$ l) $\phi\left(T^{2}\right)-\left(m \phi(T) T+n T \phi(T)+k \phi(I) T^{2}+l T^{2} \phi(I)\right) \in \mathbb{F} I$ for any $T \in \mathscr{T}$, then $\phi$ is a centralizer. And it follows that an $(m, n)-$ Jordan centralizer on a triangular algebra is a centralizer. As applications, $(m, n)$ - Jordan centralizers on (block) upper triangular matrix algebras and nest algebras are centralizers.

\section{Generalized Jordan Centralizers on Triangular Algebras}

If $\mathscr{R}$ is a unital commutative ring, $\mathscr{A}$ and $\mathscr{B}$ are unital algebras over $\mathscr{R}$ with units $1_{\mathscr{A}}$ and $1_{\mathscr{R}}$, respectively, and $\mathscr{M}$ is a unital, faithful $(\mathscr{A}, \mathscr{B})$ - bimodule, then

$$
\begin{aligned}
\mathscr{T} & =\operatorname{Tr} i(\mathscr{A}, \mathscr{M}, \mathscr{B}) \\
& =\left\{\left(\begin{array}{rr}
A & M \\
& B
\end{array}\right): A \in \mathscr{A}, M \in \mathscr{M}, B \in \mathscr{B}\right\}
\end{aligned}
$$

is a triangular algebra.

Let $\operatorname{Tr} i(\mathscr{A}, \mathscr{M}, \mathscr{B})$ be a triangular algebra. And let $P_{1}=$ $\left(\begin{array}{cc}1_{\mathscr{A}} & 0 \\ 0\end{array}\right), P_{2}=\left(\begin{array}{cc}0 & 0 \\ 1_{\mathscr{B}}\end{array}\right)$, where $1_{\mathscr{A}}$ and $1_{\mathscr{B}}$ are the identity elements of algebras $\mathscr{A}$ and $\mathscr{B}$, respectively. And let
$\mathscr{T}_{i j}=P_{i} \mathscr{T} P_{j}(1 \leq i \leq j \leq 2)$. Then $\mathscr{T}=\mathscr{T}_{11} \oplus \mathscr{T}_{12} \oplus \mathscr{T}_{22}$ and $P_{1} P_{2}=P_{2} P_{1}=0$.

The main result is as follows.

Theorem 1. Let $\mathscr{T}=\operatorname{Tri}(\mathscr{A}, \mathscr{M}, \mathscr{B})$ be a triangular algebra and $\phi: \mathscr{T} \longrightarrow \mathscr{T}$ be an additive mapping. If there exist numbers $m, n, k, l \geq 0: m n \neq 0$ such that

$$
\begin{aligned}
(m+ & n+k+l) \phi\left(T^{2}\right) \\
& \quad-\left(m \phi(T) T+n T \phi(T)+k \phi(I) T^{2}+l T^{2} \phi(I)\right) \\
& \in \mathbb{F} I
\end{aligned}
$$

for any $T=\left(\begin{array}{c}A \\ M\end{array}\right) \in \mathscr{T}$, then $\phi$ is a centralizer; that is, $\phi(I) T=$ $T \phi(I)$ and $\phi(T)=\phi(I) T=T \phi(I)$ for any $T=\left(\begin{array}{c}A M \\ B\end{array}\right) \in \mathscr{T}$, where $I=\left(\begin{array}{cc}1_{\mathscr{A}} & 0 \\ & 1_{\mathscr{B}}\end{array}\right)$.

The proof of Theorem 1 can be divided into several lemmas.

Lemma 2. Suppose that $\mathscr{T}$ is a unital algebra, and $\phi$ is an additive mapping on $\mathscr{T}$ such that

$$
\begin{aligned}
(m+ & n+k+l) \phi\left(T^{2}\right) \\
& \quad-\left(m \phi(T) T+n T \phi(T)+k \phi(I) T^{2}+l T^{2} \phi(I)\right) \\
& \in \mathbb{F} I
\end{aligned}
$$

That is, for any $T \in \mathscr{T}$, there is $\mu_{T} \in \mathbb{F}$ (depending on $T$ ) such that

$$
\begin{aligned}
(m+ & n+k+l) \phi\left(T^{2}\right) \\
= & m \phi(T) T+n T \phi(T)+k \phi(I) T^{2}+l T^{2} \phi(I) \\
& +\mu_{T} I,
\end{aligned}
$$

where $m>0, n>0, k \geq 0, l \geq 0$. Then

(1)

$$
\begin{aligned}
(m+ & n+k+l) \phi(T S+S T) \\
= & m \phi(T) S+n T \phi(S)+m \phi(S) T+n S \phi(T) \\
& +k \phi(I) T S+k \phi(I) S T+l T S \phi(I)+l S T \phi(I) \\
& +\left(\mu_{T+S}-\mu_{T}-\mu_{S}\right) I
\end{aligned}
$$

(2)

$$
\begin{aligned}
(m & +n+2 k+2 l) \phi(T) \\
& =(m+2 k) \phi(I) T+(n+2 l) T \phi(I)+\left(\mu_{T+I}-\mu_{T}\right) I
\end{aligned}
$$

for any $T, S \in \mathscr{T}$. 
Proof. For any $T, S \in \mathscr{T}$,

$$
\begin{aligned}
(m+ & n+k+l) \phi(T+S)^{2} \\
= & m \phi(T+S)(T+S)+n(T+S) \phi(T+S) \\
& +k \phi(I)(T+S)^{2}+l(T+S)^{2} \phi(I)+\mu_{T+S} I \\
= & m \phi(T) T+m \phi(T) S+m \phi(S) T+m \phi(S) S \\
& +n T \phi(T)+n T \phi(S)+n S \phi(T)+n S \phi(S) \\
& +k \phi(I) T^{2}+k \phi(I) S T+k \phi(I) T S+k \phi(I) S^{2} \\
& +l T^{2} \phi(I)+l T S \phi(I)+l S T \phi(I)+l S^{2} \phi(I) \\
& +\mu_{T+S} I .
\end{aligned}
$$

On the other hand,

$$
\begin{aligned}
(m+ & n+k+l) \phi(T+S)^{2} \\
= & (m+n+k+l) \phi\left(T^{2}+T S+S T+S^{2}\right) \\
= & m \phi(T) T+n T \phi(T)+k \phi(I) T^{2}+l T^{2} \phi(I) \\
& +n S \phi(S)+m \phi(S) S+k \phi(I) S^{2}+l S^{2} \phi(I) \\
& +(m+n+k+l) \phi(T S+S T)+\mu_{T} I+\mu_{S} I .
\end{aligned}
$$

Comparing the above two equalities, we obtain that

$$
\begin{aligned}
(m+ & n+k+l) \phi(T S+S T) \\
= & m \phi(T) S+n T \phi(S)+m \phi(S) T+n S \phi(T) \\
& +k \phi(I) T S+k \phi(I) S T+l T S \phi(I)+l S T \phi(I) \\
& +\left(\mu_{T+S}-\mu_{T}-\mu_{S}\right) I .
\end{aligned}
$$

Putting in (11) $S=I$, it follows from $\mu_{I}=0$ that

$$
\begin{aligned}
(m+ & n+2 k+2 l) \phi(T) \\
= & (m+2 k) \phi(I) T+(n+2 l) T \phi(I) \\
& +\left(\mu_{T+I}-\mu_{\mathrm{T}}\right) I .
\end{aligned}
$$

Lemma 3. Let $\mathscr{T}$ be a unital algebra and $\phi$ be an additive mapping on $\mathscr{T}$ satisfying (4). If $T_{0} \in \mathscr{T}$ with $T_{0} \notin \mathbb{F} I$ such that $T_{0} \phi(I)=\phi(I) T_{0}$, then $\mu_{T_{0}+I}-\mu_{T_{0}}=0$ and $\phi\left(T_{0}\right)=T_{0} \phi(I)=$ $\phi(I) T_{0}$.

Proof. Since $T_{0} \phi(I)=\phi(I) T_{0}, T_{0}^{2} \phi(I)=\phi(I) T_{0}^{2}=T_{0} \phi(I) T_{0}$. By (12), we have that

$$
\phi\left(T_{0}\right)=\phi(I) T_{0}+\frac{1}{m+n+2 k+2 l}\left(\mu_{T_{0}+I}-\mu_{T_{0}}\right) I
$$

and

$$
\phi\left(T_{0}^{2}\right)=\phi(I) T_{0}^{2}+\frac{1}{m+n+2 k+2 l}\left(\mu_{T_{0}^{2}+I}-\mu_{T_{0}^{2}}\right) I .
$$

Hence

$$
\begin{aligned}
(m+n+k+l) \phi\left(T_{0}^{2}\right) & \\
= & (m+n+k+l) \phi(I) T_{0}^{2} \\
& \quad+\frac{m+n+k+l}{m+n+2 k+2 l}\left(\mu_{T_{0}^{2}+I}-\mu_{T_{0}^{2}}\right) I .
\end{aligned}
$$

On the other hand,

$$
\begin{aligned}
& (m+n+k+l) \phi\left(T_{0}^{2}\right)=m \phi\left(T_{0}\right) T_{0}+n T_{0} \phi\left(T_{0}\right) \\
& \quad+k \phi(I) T_{0}^{2}+l T_{0}^{2} \phi(I)+\mu_{T_{0}} I \\
& \quad=m\left(\phi(I) T_{0}+\frac{1}{m+n+2 k+2 l}\left(\mu_{T_{0}+I}-\mu_{T_{0}}\right) I\right) \\
& \quad \cdot T_{0} \\
& \quad+n T_{0}\left(\phi(I) T_{0}+\frac{1}{m+n+2 k+2 l}\left(\mu_{T_{0}+I}-\mu_{T_{0}}\right) I\right) \\
& \quad+k \phi(I) T_{0}^{2}+l T_{0}^{2} \phi(I)+\mu_{T_{0}} I .
\end{aligned}
$$

Comparing the two equalities, we have that $((m+n) /(m+n+$ $2 k+2 l))\left(\mu_{T_{0}+I}-\mu_{T_{0}}\right) T_{0} \in \mathbb{F} I$. Since $T_{0} \notin \mathbb{F} I$ and $m+n>0$, $\mu_{T_{0}+I}-\mu_{T_{0}}=0$ and $\phi\left(T_{0}\right)=T_{0} \phi(I)=\phi(I) T_{0}$.

Lemma 4. Let $\mathscr{T}$ be a unital algebra and $\phi$ be an additive mapping on $\mathscr{T}$ satisfying (4). If $P \in \mathscr{T}$ with $P^{2}=P$, then $\phi(P)=P \phi(I)=\phi(I) P=\phi(P) P=P \phi(P)$.

Proof. If $P=0$ or $P=I$, the result is trivial.

Let $P$ be a nontrivial idempotent; that is, $P \neq 0$ and $P \neq I$. By (4),

$$
\begin{aligned}
(m+n+k+l) \phi(P)= & m \phi(P) P+n P \phi(P) \\
& +k \phi(I) P+l P \phi(I)+\mu_{P} I .
\end{aligned}
$$

By (12),

$$
\begin{aligned}
(m+ & n+2 k+2 l) \phi(P) \\
= & (m+2 k) \phi(I) P+(n+2 l) P \phi(I) \\
& +\left(\mu_{P+I}-\mu_{P}\right) I .
\end{aligned}
$$

Multiplying (17) by $P$ from the left and the right sides gives

$$
(k+l) P \phi(P) P=(k+l) P \phi(I) P+\mu_{P} P .
$$

Multiplying (18) by $P$ from the left and the right sides, we have that

$$
P \phi(P) P=P \phi(I) P+\frac{1}{m+n+2 k+2 l}\left(\mu_{P+I}-\mu_{P}\right) P .
$$

By comparing the above two equalities,

$$
(m+n+2 k+2 l) \mu_{P}=(k+l)\left(\mu_{P+I}-\mu_{P}\right) .
$$


Multiplying (17) by $P$ from the left side gives that

$$
\begin{aligned}
&(m+n+k+l) P \phi(P) \\
&= m P \phi(P) P+n P \phi(P)+k P \phi(I) P+l P \phi(I) \\
&+\mu_{P} P .
\end{aligned}
$$

That is,

$$
\begin{aligned}
(m+k+l) P \phi(P)= & m P \phi(P) P+k P \phi(I) P+l P \phi(I) \\
& +\mu_{P} P .
\end{aligned}
$$

It follows from (20) that

$$
\begin{aligned}
(m+ & k+l) P \phi(P) \\
= & (m+k) P \phi(I) P+l P \phi(I) \\
& \quad+\left(\frac{m}{m+n+2 k+2 l}\left(\mu_{P+I}-\mu_{P}\right)+\mu_{P}\right) P .
\end{aligned}
$$

Thus

$$
\begin{aligned}
(m+ & n+2 k+2 l)(m+k+l) P \phi(P) \\
= & (m+k)(m+n+2 k+2 l) P \phi(I) P \\
& +l(m+n+2 k+2 l) P \phi(I) \\
& +\left(m\left(\mu_{P+I}-\mu_{P}\right)+(m+n+2 k+2 l) \mu_{P}\right) P .
\end{aligned}
$$

Multiplying (18) by $P$ from the left side yields that

$$
\begin{aligned}
(m+ & n+2 k+2 l) P \phi(P) \\
= & (m+2 k) P \phi(I) P+(n+2 l) P \phi(I) \\
& +\left(\mu_{P+I}-\mu_{P}\right) P,
\end{aligned}
$$

and comparing $(24)^{\prime}$ and (25), we obtain that

$$
\begin{aligned}
(m+ & k+l)(m+2 k) P \phi(I) P \\
& +(n+2 l)(m+k+l) P \phi(I) \\
& +(m+k+l)\left(\mu_{P+I}-\mu_{P}\right) P \\
= & (m+k)(m+n+2 k+2 l) P \phi(I) P \\
& +l(m+n+2 k+2 l) P \phi(I) \\
& +\left(m\left(\mu_{P+I}-\mu_{P}\right)+(m+n+2 k+2 l) \mu_{P}\right) P .
\end{aligned}
$$

It follows from $(21)$ that $(m+k+l)\left(\mu_{P+I}-\mu_{P}\right) P=\left(m\left(\mu_{P+I}-\right.\right.$ $\left.\left.\mu_{P}\right)+(m+n+2 k+2 l) \mu_{P}\right) P$ and

$$
P \phi(I)=P \phi(I) P .
$$

It follows from (24) that

$$
P \phi(P)=P \phi(I) P+\frac{1}{m+n+2 k+2 l}\left(\mu_{P+I}-\mu_{P}\right) P .
$$

Similarly,

$$
\phi(I) P=P \phi(I) P
$$

and

$$
\phi(P) P=P \phi(I) P+\frac{1}{m+n+2 k+2 l}\left(\mu_{P+I}-\mu_{P}\right) P .
$$

Equations (27) and (29) yield that $\phi(I) P=P \phi(I)$. And

$$
\phi(P) P=P \phi(P)=P \phi(P) P
$$

by (28) and (30). By Lemma 3 and $\phi(I) P=P \phi(I)$, it follows that $\phi(P)=\phi(I) P=P \phi(I)$ and $\mu_{P+I}-\mu_{P}=0$. And by (21), $\mu_{P}=0$ and $\mu_{P+I}=\mu_{P}=0$. Identity (17) yields that

$$
\begin{aligned}
\phi(P) & =\phi(I) P=P \phi(I)=P \phi(I) P=P \phi(P) P \\
& =\phi(P) P=P \phi(P) .
\end{aligned}
$$

Lemma 5. Let $\mathscr{T}$ be a unital algebra and $\phi$ be an additive mapping on $\mathscr{T}$ satisfying (4). If T, $P \in \mathscr{A}$ with $P^{2}=P$, then

(1) $\phi(T P)=\phi(T) P+\mu(T P) I-\mu(T) P$,

(2) $\phi(P T)=P \phi(T)+\mu(P T) I-\mu(T) P$, where $\mu(T)=$ $(1 /(m+n+2 k+2 l))\left(\mu_{T+I}-\mu_{T}\right)$.

Proof. By (12),

$$
\begin{aligned}
\phi(T P) & =\frac{m+2 k}{m+n+2 k+2 l} \phi(I) T P+\frac{n+2 l}{m+n+2 k+2 l} \\
\cdot & T P \phi(I)+\frac{1}{m+n+2 k+2 l}\left(\mu_{T P+I}-\mu_{T P}\right) I \\
& =\left(\frac{m+2 k}{m+n+2 k+2 l} \phi(I) T\right. \\
& \left.+\frac{n+2 l}{m+n+2 k+2 l} T \phi(I)\right) P \\
& +\frac{1}{m+n+2 k+2 l}\left(\mu_{T P+I}-\mu_{T P}\right) I=\phi(T) P \\
& +\mu(T P) I-\mu(T) P .
\end{aligned}
$$

Similarly, $\phi(P T)=P \phi(T)+\mu(P T) I-\mu(T) P$.

Lemma 6. Let $\mathscr{T}$ be a unital algebra and $\phi$ be an additive mapping on $\mathscr{T}$ satisfying (4). If T, $P \in \mathscr{T}$ with $P^{2}=P$, then $\phi(P T P)=\phi(P T P) P=P \phi(P T P)=P \phi(P T P) P$.

Proof. If $P=0$ or $P=I$, the result is trivial.

Let $P$ be a nontrivial idempotent; that is, $P \neq 0$ and $P \neq I$. It follows from Lemma 5 that

$$
\begin{aligned}
\phi(P T P) & =\phi(P T P P) \\
& =\phi(P T P) P+\mu(P T P) I-\mu(P T P) P, \\
\phi(P T P) & =\phi(P P T P) \\
& =P \phi(P T P)+\mu(P T P) I-\mu(P T P) P .
\end{aligned}
$$

Comparing the above two equalities, we have that

$$
P \phi(P T P)=\phi(P T P) P \text {. }
$$


It follows from Lemma 2(2) that

$$
\begin{aligned}
(m+ & n+2 k+2 l) \phi(P T P) \\
= & (m+2 k) \phi(I) P T P+(n+2 l) P T P \phi(I) \\
& +\left(\mu_{P T P+I}-\mu_{P T P}\right) I .
\end{aligned}
$$

By Lemma 4, we have that $\phi(I) P T P=\phi(P) P T P, P T P \phi(I)=$ $P T P \phi(P)$, and $\mu_{P}=0$. Putting $P T P$ for $T$ and $P$ for $S$ in (11), we have that

$$
\begin{aligned}
2(m+n+k+l) \phi(P T P) \\
=(m+n+k+l) \phi((P T P) P+P(P T P)) \\
=m \phi(P T P) P+n P \phi(P T P)+n P T P \phi(P) \\
\quad+m \phi(P) P T P+2 k \phi(I) P T P+2 l P T P \phi(I) \\
\quad+\left(\mu_{P T P+P}-\mu_{P T P}-\mu_{P}\right) I \\
=(m+n) P \phi(P T P)+(m+2 k) \phi(I) P T P \\
\quad+(n+2 l) P T P \phi(I)+\left(\mu_{P T P+P}-\mu_{P T P}\right) I .
\end{aligned}
$$

By (36) with (37),

$$
\begin{aligned}
& 2(m+n+k+l)(m+n+2 k+2 l) \phi(P T P)=(m \\
& \quad+n) P((m+2 k) \phi(I) P T P+(n+2 l) P T P \phi(I) \\
& \left.+\left(\mu_{P T P+I}-\mu_{P T P}\right) I\right)+(m+2 k)(m+n+2 k+2 l) \\
& \quad \phi(I) P T P+(n+2 l)(m+n+2 k+2 l) P T P \phi(I) \\
& \quad+(m+n+2 k+2 l)\left(\mu_{P T P+P}-\mu_{P T P}\right) I=2(m \\
& \quad+2 k)(m+n+k+l) \phi(I) P T P+2(n+2 l)(m+n \\
& \quad+k+l) P T P \phi(I)+(m+n+2 k+2 l)\left(\mu_{P T P+P}\right. \\
& \left.\quad-\mu_{P T P}\right) I+(m+n)\left(\mu_{P T P+I}-\mu_{P T P}\right) P .
\end{aligned}
$$

It follows from (36) that

$$
\begin{aligned}
& 2(m+n+k+l)(m+n+2 k+2 l) \phi(P T P) \\
&= 2(m+2 k)(m+n+k+l) \phi(I) P T P \\
&+2(n+2 l)(m+n+k+l) P T P \phi(I) \\
&+2(m+n+k+l)\left(\mu_{P T P+I}-\mu_{P T P}\right) I .
\end{aligned}
$$

Comparing (38) and (39), we have that

$$
\begin{gathered}
(m+n+2 k+2 l)\left(\mu_{P T P+P}-\mu_{P T P}\right) I \\
+(m+n)\left(\mu_{P T P+I}-\mu_{P T P}\right) P \\
=2(m+n+k+l)\left(\mu_{P T P+I}-\mu_{P T P}\right) I .
\end{gathered}
$$

Multiplying (36) by $P$ from the left and the right sides gives that

$$
\begin{aligned}
(m+ & n+2 k+2 l) P \phi(P T P) P \\
= & (m+2 k) \phi(I) P T P+(n+2 l) P T P \phi(I) \\
& +\left(\mu_{P T P+I}-\mu_{P T P}\right) P .
\end{aligned}
$$

Multiplying (37) by $P$ from both sides yields that

$$
\begin{aligned}
& 2(m+n+k+l) P \phi(P T P) P \\
&=(m+n) P \phi(P T P) P+(m+2 k) \phi(I) P T P \\
&+(n+2 l) P T P \phi(I)+\left(\mu_{P T P+P}-\mu_{P T P}\right) P .
\end{aligned}
$$

It follows that

$$
\begin{aligned}
(m+ & n+2 k+2 l) P \phi(P T P) P \\
= & (m+2 k) \phi(I) P T P+(n+2 l) P T P \phi(I) \\
& +\left(\mu_{P T P+P}-\mu_{P T P}\right) P .
\end{aligned}
$$

Comparing (41) and (43), we have that

$$
\mu_{P T P+P}-\mu_{P T P}=\mu_{P T P+I}-\mu_{P T P}
$$

It follows from the above equality and (40) that $(m+$ $n)\left(\mu_{P T P+P}-\mu_{P T P}\right)=0$. Since $m+n>0, \mu_{P T P+P}-\mu_{P T P}=0$ and

$$
\mu_{P T P+P}-\mu_{P T P}=\mu_{P T P+I}-\mu_{P T P}=0 .
$$

By (37) and (45),

$$
\begin{aligned}
& 2(m+n+k+l) \phi(P T P) \\
&=(m+n) P \phi(P T P)+(m+2 k) \phi(I) P T P \\
&+(n+2 l) P T P \phi(I) .
\end{aligned}
$$

By (36) and (45),

$$
\begin{aligned}
(m & +n+2 k+2 l) \phi(P T P) \\
& =(m+2 k) \phi(I) P T P+(n+2 l) P T P \phi(I) .
\end{aligned}
$$

Comparing it with (46), we have that $(m+n) \phi(P T P)=(m+$ n) $P \phi(P T P)$ and

$$
\phi(P T P)=P \phi(P T P)=\phi(P T P) P=P \phi(P T P) P .
$$

Lemma 7. Let $\mathscr{T}=\operatorname{Tri}(\mathscr{A}, \mathscr{M}, \mathscr{B})$ be a triangular algebra. Suppose that $T_{i j} \in \mathscr{T}_{i j}(1 \leq i \leq j \leq 2)$.

(1) If $T_{11} \mathscr{T}_{12}=0$, then $T_{11}=0$;

(2) If $\mathscr{T}_{12} T_{22}=0$, then $T_{22}=0$.

Proof. (1) If $T_{11} \mathscr{T}_{12}=0$ and $T_{11}=\left(\begin{array}{cc}1_{\mathscr{A}} & 0 \\ 0\end{array}\right)\left(\begin{array}{cc}A_{0} & M_{0} \\ B_{0}\end{array}\right)\left(\begin{array}{cc}1_{\mathscr{A}} & 0 \\ & 0\end{array}\right)$, then $\left(\begin{array}{ll}A_{0} & 0 \\ & 0\end{array}\right)\left(\begin{array}{cc}1_{\mathscr{A}} & 0 \\ & 0\end{array}\right)\left(\begin{array}{cc}A & M \\ B\end{array}\right)\left(\begin{array}{cc}0 & 0 \\ & 1_{\mathscr{B}}\end{array}\right)=0$ for any $A \in \mathscr{A}, M \in$ $\mathscr{M}, B \in \mathscr{B}$. So $A_{0} M=0$ for any $M \in \mathscr{M}$. It follows from the definition of a triangular algebra that $A_{0}=0$. And $T_{11}=$ $\left(\begin{array}{cc}1_{\mathscr{A}} & 0 \\ 0\end{array}\right)\left(\begin{array}{cc}A_{0} & M_{0} \\ B_{0}\end{array}\right)\left(\begin{array}{cc}1_{\mathscr{A}} & 0 \\ & 0\end{array}\right)=0$.

(2) It is similar to the proof of (1).

Lemma 8. Let $\mathscr{T}=\operatorname{Tri}(\mathscr{A}, \mathscr{M}, \mathscr{B})$ be a triangular algebra, and $\phi: \mathscr{T} \longrightarrow \mathscr{T}$ be an additive mapping satisfying (4). Then $\phi\left(\mathscr{T}_{i j}\right) \subseteq \mathscr{T}_{i j}(1 \leq i \leq j \leq 2)$. 
Proof. It follows that $\phi\left(\mathscr{T}_{11}\right) \subseteq \mathscr{T}_{11}$ and $\phi\left(\mathscr{T}_{22}\right) \subseteq \mathscr{T}_{22}$ from Lemma 6.

For any $T \in \mathscr{T}$, by Lemma 5 , we have that

$$
\begin{aligned}
\phi\left(P_{1} T P_{2}\right)= & \phi\left(P_{1} T P_{2} P_{2}\right) \\
= & \phi\left(P_{1} T P_{2}\right) P_{2}+\mu\left(P_{1} T P_{2} P\right) I \\
& -\mu\left(P_{1} T P_{2}\right) P_{2} \\
= & \phi\left(P_{1} T P_{2}\right) P_{2}+\mu\left(P_{1} T P_{2} P\right) P_{1}
\end{aligned}
$$

and

$$
\begin{aligned}
\phi\left(P_{1} T P_{2}\right)= & \phi\left(P_{1} P_{1} T P_{2}\right) \\
= & P_{1} \phi\left(P_{1} T P_{2}\right)+\mu\left(P_{1} T P_{2} P\right) I \\
& -\mu\left(P_{1} T P_{2}\right) P_{1} \\
= & P_{1} \phi\left(P_{1} T P_{2}\right)+\mu\left(P_{1} T P_{2} P\right) P_{2} .
\end{aligned}
$$

It follows that

$$
\phi\left(P_{1} T P_{2}\right) P_{1}=\mu\left(P_{1} T P_{2} P\right) P_{1}
$$

and

$$
P_{2} \phi\left(P_{1} T P_{2}\right)=\mu\left(P_{1} T P_{2} P\right) P_{2}
$$

By (12), we have that

$$
\begin{aligned}
(m+ & n+2 k+2 l) \phi\left(P_{1} T P_{2}\right) \\
= & (m+2 k) \phi(I) P_{1} T P_{2}+(n+2 l) P_{1} T P_{2} \phi(I) \\
& +\left(\mu_{P_{1} T P_{2}+I}-\mu_{P_{1} T P_{2}}\right) I .
\end{aligned}
$$

Multiplying the above equality by $P_{1}$ from the right side gives that

$$
\begin{aligned}
& (m+n+2 k+2 l) \phi\left(P_{1} T P_{2}\right) P_{1} \\
& \quad=\left(\mu_{P_{1} T P_{2}+I}-\mu_{P_{1} T P_{2}}\right) P_{1}=\mu\left(P_{1} T P_{2} P\right) P_{1} .
\end{aligned}
$$

It follows that

$$
\begin{aligned}
& (m+n+2 k+2 l) \phi\left(P_{1} T P_{2}\right) P_{1}=\mu\left(P_{1} T P_{2} P\right) P_{1} \\
& \quad=\phi\left(P_{1} T P_{2}\right) P_{1}
\end{aligned}
$$

and

$$
\phi\left(P_{1} T P_{2}\right) P_{1}=0
$$

Similarly, we have that

$$
P_{2} \phi\left(P_{1} T P_{2}\right)=0
$$

It follows that

$$
\phi\left(P_{1} T P_{2}\right)=P_{1} \phi\left(P_{1} T P_{2}\right)=\phi\left(P_{1} T P_{2}\right) P_{2}
$$

and $\phi\left(\mathscr{T}_{12}\right) \subseteq \mathscr{T}_{12}$.
Lemma 9. Let $\mathscr{T}=\operatorname{Tri}(\mathscr{A}, \mathscr{M}, \mathscr{B})$ be a triangular algebra, and $\phi: \mathscr{T} \longrightarrow \mathscr{T}$ be an additive mapping satisfying (4). Let $T_{i j}, S_{i j} \in \mathscr{T}_{i j}(1 \leq i \leq j \leq 2)$. Then
(1) $\phi\left(T_{11} S_{12}\right)=\phi\left(T_{11}\right) S_{12}=T_{11} \phi\left(S_{12}\right)$;
(2) $\phi\left(T_{12} S_{22}\right)=\phi\left(T_{12}\right) S_{22}=T_{12} \phi\left(S_{22}\right)$;
(3) $\phi\left(T_{11} S_{11}\right)=\phi\left(T_{11}\right) S_{11}=T_{11} \phi\left(S_{11}\right)$;
(4) $\phi\left(T_{22} S_{22}\right)=\phi\left(T_{22}\right) S_{22}=T_{22} \phi\left(S_{22}\right)$.

Proof. (1) By (11),

$$
\begin{aligned}
(m+ & n+k+l) \phi\left(T_{11} S_{12}+S_{12} T_{11}\right) \\
= & m \phi\left(T_{11}\right) S_{12}+n T_{11} \phi\left(S_{12}\right)+m \phi\left(S_{12}\right) T_{11} \\
& +n S_{12} \phi\left(T_{11}\right)+k \phi(I) T_{11} S_{12}+k \phi(I) S_{12} T_{11} \\
& +l T_{11} S_{12} \phi(I)+l S_{12} T_{11} \phi(I) \\
& +\left(\mu_{T_{11}+S_{12}}-\mu_{T_{11}}-\mu_{S_{12}}\right) I .
\end{aligned}
$$

Since $S_{12} T_{11}=0, \phi\left(S_{12}\right) T_{11} \in \mathscr{T}_{12} T_{11}=0$, and $S_{12} \phi\left(T_{11}\right) \in$ $S_{12} \mathscr{T}_{11}=0$, it follows that

$$
\begin{aligned}
(m+ & n+k+l) \phi\left(T_{11} S_{12}\right) \\
= & m \phi\left(T_{11}\right) S_{12}+n T_{11} \phi\left(S_{12}\right)+k \phi(I) T_{11} S_{12} \\
& +l T_{11} S_{12} \phi(I)+\left(\mu_{T_{11}+S_{12}}-\mu_{T_{11}}-\mu_{S_{12}}\right) I .
\end{aligned}
$$

Multiplying (60) by $P_{1}$ from the right side, using the fact that $\phi\left(S_{12}\right), \phi\left(T_{11} S_{12}\right) \in \mathscr{T}_{12}$, yields that $\left(\mu_{T_{11}+S_{12}}-\mu_{T_{11}}-\mu_{S_{12}}\right) P_{1}=0$ and $\mu_{T_{11}+S_{12}}-\mu_{T_{11}}-\mu_{S_{12}}=0$. Since $S_{12} \in \mathscr{T}_{12}$, it follows from Lemma 7(2) that

$$
\phi\left(S_{12}\right)=\phi(I) S_{12}=S_{12} \phi(I) .
$$

Since $T_{11} S_{12} \in \mathscr{T}_{12}$,

$$
\phi\left(T_{11} S_{12}\right)=\phi(I) T_{11} S_{12}=T_{11} S_{12} \phi(I)=T_{11} \phi\left(S_{12}\right) .
$$

Combining it with (60), we have that $m \phi\left(T_{11} S_{12}\right)=$ $m \phi\left(T_{11}\right) S_{12}$. Since $m \neq 0$,

$$
\begin{aligned}
\phi\left(T_{11}\right) S_{12} & =T_{11} S_{12} \phi(I)=\phi\left(T_{11} S_{12}\right)=T_{11} \phi\left(S_{12}\right) \\
& =\phi(I) T_{11} S_{12} .
\end{aligned}
$$

(2) By (11),

$$
\begin{aligned}
(m+ & n+k+l) \phi\left(T_{12} S_{22}+S_{22} T_{12}\right) \\
= & m \phi\left(T_{12}\right) S_{22}+n T_{12} \phi\left(S_{22}\right)+m \phi\left(S_{22}\right) T_{12} \\
& +n S_{22} \phi\left(T_{12}\right)+k \phi(I) T_{12} S_{22}+k \phi(I) S_{22} T_{12} \\
& +l T_{12} S_{22} \phi(I)+l S_{22} T_{12} \phi(I) \\
& +\left(\mu_{T_{12}+S_{22}}-\mu_{T_{12}}-\mu_{S_{22}}\right) I .
\end{aligned}
$$


Using the fact that $\phi\left(S_{22}\right) T_{12}, S_{22} \phi\left(T_{12}\right) \in \mathscr{T}_{22} \mathscr{T}_{12}=0$ yields that

$$
\begin{aligned}
(m+ & n+k+l) \phi\left(T_{12} S_{22}\right) \\
= & m \phi\left(T_{12}\right) S_{22}+n T_{12} \phi\left(S_{22}\right)+k \phi(I) T_{12} S_{22} \\
& +l T_{12} S_{22} \phi(I)+\left(\mu_{T_{12}+S_{22}}-\mu_{T_{12}}-\mu_{S_{22}}\right) I .
\end{aligned}
$$

Multiplying (65) by $P_{1}$ from the right side, we have that $\left(\mu_{T_{12}+S_{22}}-\mu_{T_{12}}-\mu_{S_{22}}\right) P_{1}=0$ and $\mu_{T_{12}+S_{22}}-\mu_{T_{12}}-\mu_{S_{22}}=0$. It follows from Lemma $7(2)$ that $\phi\left(T_{12}\right)=\phi(I) T_{12}=T_{12} \phi(I)$ and

$$
\phi\left(T_{12} S_{22}\right)=T_{12} S_{22} \phi(I)=\phi(I) T_{12} S_{22}=\phi\left(T_{12}\right) S_{22} .
$$

Combining it with (65), we have that $n \phi\left(T_{12} S_{22}\right)=$ $n T_{12} \phi\left(S_{22}\right)$. Since $n \neq 0$,

$$
\begin{aligned}
\phi\left(T_{12} S_{22}\right) & =\phi\left(T_{12}\right) S_{22}=T_{12} \phi\left(S_{22}\right)=\phi(I) T_{12} S_{22} \\
& =T_{12} S_{22} \phi(I) .
\end{aligned}
$$

(3) By (1), for any $R_{12} \in \mathscr{T}_{12}$,

$$
\phi\left(T_{11} S_{11}\right) R_{12}=\phi\left(T_{11} S_{11} R_{12}\right)=\phi\left(T_{11}\right) S_{11} R_{12},
$$

and

$$
\begin{aligned}
\phi\left(T_{11} S_{11}\right) R_{12} & =\phi\left(T_{11} S_{11} R_{12}\right)=T_{11} \phi\left(S_{11} R_{12}\right) \\
& =T_{11} \phi\left(S_{11}\right) R_{12} .
\end{aligned}
$$

$\phi\left(T_{11} S_{11}\right)=\phi\left(T_{11}\right) S_{11}=T_{11} \phi\left(S_{11}\right)$ by Lemma $7(1)$.

(4) By (2), for any $R_{12} \in \mathscr{T}_{12}$,

$$
R_{12} \phi\left(T_{22} S_{22}\right)=\phi\left(R_{12} T_{22} S_{22}\right)=R_{12} T_{22} \phi\left(S_{22}\right),
$$

and

$$
\begin{aligned}
R_{12} \phi\left(T_{22} S_{22}\right) & =\phi\left(R_{12} T_{22} S_{22}\right)=\phi\left(R_{12} T_{22}\right) S_{22} \\
& =R_{12} \phi\left(T_{22}\right) S_{22} .
\end{aligned}
$$

$\phi\left(T_{22} S_{22}\right)=\phi\left(T_{22}\right) S_{22}=T_{22} \phi\left(S_{22}\right)$ by Lemma $7(2)$.

Proof of Theorem 1. Let $T, S \in \mathscr{T}$. Then $T=T_{11}+T_{12}+T_{22}$ and $S=S_{11}+S_{12}+S_{22}$. By Lemmas 8 and 9 , we have that

$$
\begin{aligned}
\phi(T S)= & \phi\left(T_{11} S_{11}+T_{11} S_{12}+T_{12} S_{22}+T_{22} S_{22}\right) \\
= & \phi\left(T_{11} S_{11}\right)+\phi\left(T_{11} S_{12}\right)+\phi\left(T_{12} S_{22}\right) \\
& +\phi\left(T_{22} S_{22}\right) \\
= & \phi\left(T_{11}\right) S_{11}+\phi\left(T_{11}\right) S_{12}+\phi\left(T_{12}\right) S_{22} \\
& +\phi\left(T_{22}\right) S_{22} \\
= & T_{11} \phi\left(S_{11}\right)+T_{11} \phi\left(S_{12}\right)+T_{12} \phi\left(S_{22}\right) \\
& +T_{22} \phi\left(S_{22}\right) \\
= & \phi\left(T_{11}+T_{12}+T_{22}\right)\left(S_{11}+S_{12}+S_{22}\right) \\
= & \left(T_{11}+T_{12}+T_{22}\right) \phi\left(S_{11}+S_{12}+S_{22}\right) \\
= & \phi(T) S=T \phi(S) .
\end{aligned}
$$

So that $\phi$ is a centralizer.
Corollary 10. Let $\mathscr{T}=\operatorname{Tri}(\mathscr{A}, \mathscr{M}, \mathscr{B})$ be a triangular algebra, and $\phi: \mathscr{T} \longrightarrow \mathscr{T}$ be an additive mapping. If there are positive numbers $m, n$, such that $(m+n) \phi\left(T^{2}\right)=m \phi(T) T+n T \phi(T)$ for any $T \in \mathscr{T}$, then $\phi$ is a centralizer.

Theorem 11. Let $\mathscr{T}=\operatorname{Tri}(\mathscr{A}, \mathscr{M}, \mathscr{B})$ be a triangular algebra, and $\phi: \mathscr{T} \longrightarrow \mathscr{T}$ be an additive mapping. If there are positive numbers $m, n$ and a positive integer $p$, such that

$$
(m+n) \phi\left(T^{p+1}\right)=m \phi(T) T^{p}+n T^{p} \phi(T)
$$

for any $T \in \mathscr{T}$, then $\phi$ is a centralizer.

Proof. Putting $T+x I$ for $T$ in (73), where $x$ is any rational number, we get that $(m+n) \phi\left(T^{2}\right)=m \phi(T) T+n T \phi(T)$ for any $T \in \mathscr{T}$, so that $\phi$ is a centralizer by Corollary 10 .

Theorem 12. Let $\mathscr{T}=\operatorname{Tri}(\mathscr{A}, \mathscr{M}, \mathscr{B})$ be a triangular algebra and $\phi: \mathscr{T} \longrightarrow \mathscr{T}$ be an additive mapping. If there are positive integers $m, n$, such that

$$
\phi\left(T^{m+n+1}\right)=T^{m} \phi(T) T^{n}
$$

for any $T \in \mathscr{T}$, then $\phi$ is a centralizer.

Proof. Putting $T+x I$ for $T$ in (74), where $x$ is any rational number, we can also get that $(m+n) \phi\left(T^{2}\right)=m \phi(T) T+n T \phi(T)$ for any $T \in \mathscr{T}$, so that $\phi$ is a centralizer by Corollary 10 .

\section{Applications}

Upper triangular matrix algebras, block upper triangular matrix algebras, and nest algebras over a Hilbert space $H$ are important examples of triangular algebras.

Upper triangular matrix algebras. Let $\mathscr{M}_{k \times m}(R)$ be the set of all $k \times m$ matrices and $\mathscr{T}_{k}(R)$ be the algebra of all $k \times k$ upper triangular matrices over a ring $R$. For $k \geq$ 2 and each $1 \leq l \leq k-1$, the algebra $\mathscr{T}_{k}(R)$ can be represented as a triangular algebra of the form $\mathscr{T}_{k}(R)=$ $\operatorname{Tri}\left(\mathscr{T}_{l}(R), \mathscr{M}_{l \times(k-l)}(R), \mathscr{T}_{k-l}(R)\right)$.

Block upper triangular matrix algebras. Let $\mathbb{N}$ be the set of all positive integers and let $k \in \mathbb{N}$. For every positive integer $s$, $s \leq k$, we denote by $\bar{l}=\left(l_{1}, l_{2}, \ldots, l_{s}\right) \in \mathbb{N}^{s}$ an ordered $s$-vector of positive integers such that $k=l_{1}+l_{2}+\ldots+l_{s}$. The block upper triangular matrix algebra $\mathscr{B}_{k}(R)$ with corresponding vector $\bar{l}$ is a subalgebra of $\mathscr{T}_{k}(R)$ which contains all block upper triangular matrices, where diagonal blocks have sizes $l_{1}, l_{2}, \ldots, l_{s}$, respectively. Note that $\mathscr{M}_{k}(R)$ and $\mathscr{T}_{k}(R)$ are two special cases of block upper triangular matrix algebras. If we have that $k \geq 2$ and $\mathscr{B}_{k}(R) \neq \mathscr{M}_{k}(R)$, then $\mathscr{B}_{k}(R)$ is a triangular algebra and can be represented as $\mathscr{B}_{k}(R)=$ $\operatorname{Tri}\left(\mathscr{B}_{l}(R), \mathscr{M}_{l \times(k-l)}(R), \mathscr{B}_{k-l}(R)\right)$, where $1 \leq l \leq k-1$ and $\mathscr{B}_{l}(R)$ and $\mathscr{B}_{k-l}(R)$ are block upper triangular algebras with suitable vectors $\overline{l_{1}} \in \mathbb{N}^{l}, \overline{l_{2}} \in \mathbb{N}^{k-l}$.

Corollary 13. Every $(m, n)$-Jordan centralizer of a block upper triangular matrix algebra $\mathscr{B}_{k}(\mathscr{C})(k \geq 2)$ is a centralizer, where $\mathscr{C}$ is the complex field. 
Corollary 14. Let $\mathscr{B}_{k}(\mathscr{C})$ be a block upper triangular matrix algebra and $\phi: \mathscr{B}_{k}(\mathscr{C}) \longrightarrow \mathscr{B}_{k}(\mathscr{C})$ be an additive mapping on $\mathscr{B}_{k}(\mathscr{C})(k \geq 2)$. If there are positive numbers $m, n$ and a positive integer $p$, such that

$$
(m+n) \phi\left(T^{p+1}\right)=m \phi(T) T^{p}+n T^{p} \phi(T)
$$

for any $T \in \mathscr{B}_{k}(\mathscr{C})$, then $\phi$ is a centralizer.

Corollary 15. Let $\mathscr{B}_{k}(\mathscr{C})$ be a block upper triangular matrix algebra and $\phi: \mathscr{B}_{k}(\mathscr{C}) \longrightarrow \mathscr{B}_{k}(\mathscr{C})$ be an additive mapping on $\mathscr{B}_{k}(\mathscr{C})(k \geq 2)$. If there are positive numbers $m, n$ and a positive integer $p$, such that

$$
\phi\left(T^{m+n+1}\right)=T^{m} \phi(T) T^{n}
$$

for any $T \in \mathscr{B}_{k}(\mathscr{C})$, then $\phi$ is a centralizer.

Nest algebras. A nest is a chain $\mathcal{N}$ of closed subspaces of a real or complex Hilbert space $H$ containing $\{0\}$ and $H$, which is closed under arbitrary intersections and closed liner span. The nest algebra associated with $\mathcal{N}$ is the algebra

$$
\begin{aligned}
\mathscr{T} & (\mathcal{N}) \\
& =\{T: T \in B(H) \text { and } T N \subseteq N \text { for all } N \in \mathcal{N}\} .
\end{aligned}
$$

A nest $\mathcal{N}$ is called trivial if $\mathcal{N}=\{0, H\}$. A nontrivial nest algebra over a Hilbert space is a triangular algebra.

In fact, by Peirce decomposition, a nontrivial nest algebra is a triangular algebra. Suppose that $\mathscr{A}=\mathscr{T}(\mathscr{N})$ is a nontrivial nest algebra, and $\mathcal{N}$ is the corresponding nest. Choose any $P_{1} \in \mathcal{N}$ such that $P_{1} \neq 0, P_{1} \neq I$. Set $P_{2}=I-P_{1}, \mathscr{A}_{11}=P_{1} \mathscr{A} P_{1}$, $\mathscr{A}_{12}=P_{1} \mathscr{A} P_{2}$, and $\mathscr{A}_{22}=P_{2} \mathscr{A} P_{2}$. Then $\mathscr{A}=\mathscr{A}_{11} \oplus \mathscr{A}_{12} \oplus \mathscr{A}_{22}$; that is,

$$
\mathscr{A}=\left(\begin{array}{ll}
\mathscr{A}_{11} & \mathscr{A}_{12} \\
& \mathscr{A}_{22} \cdot
\end{array}\right)
$$

Since $\mathscr{A}_{12}$ is a faithful $\left(\mathscr{A}_{11}, \mathscr{A}_{22}\right)$ - bimodule, the nest algebra $\mathscr{A}$ is a triangular algebra.

Corollary 16. Let $\mathscr{T}(\mathcal{N})$ be a nontrivial nest algebra over a Hilbert space. Then every $(m, n)$-Jordan centralizer on the nest algebra $\mathscr{T}(\mathcal{N})$ is a centralizer.

Corollary 17 (see [9]). Let $\mathscr{T}(\mathcal{N})$ be a nontrivial nest algebra over a Hilbert space and let $\phi: \mathscr{T}(\mathcal{N}) \longrightarrow \mathscr{T}(\mathcal{N})$ be an additive mapping on $\mathscr{T}(\mathcal{N})$. If there are positive numbers $m, n$ and a positive integer $p$, such that

$$
(m+n) \phi\left(T^{p+1}\right)=m \phi(T) T^{p}+n T^{p} \phi(T)
$$

for any $T \in \mathscr{T}(\mathcal{N})$, then $\phi$ is a centralizer.

Corollary 18 (see [9]). Let $\mathscr{T}(\mathcal{N})$ be a nontrivial nest algebra over a Hilbert space and let $\phi: \mathscr{T}(\mathcal{N}) \longrightarrow \mathscr{T}(\mathcal{N})$ be an additive mapping on $\mathscr{T}(\mathscr{N})$. If there are positive numbers $m, n$ and a positive integer $p$, such that

$$
\phi\left(T^{m+n+1}\right)=T^{m} \phi(T) T^{n}
$$

for any $T \in \mathscr{T}(\mathcal{N})$, then $\phi$ is a centralizer.

\section{Data Availability}

No data were used to support this study.

\section{Conflicts of Interest}

The authors declare that there are no conflicts of interest regarding the publication of this paper.

\section{Acknowledgments}

The authors are supported by the National Science Foundation of China (No. 11401273, No. 11871375) and the Natural Science Research Foundation of Jiangxi Provincial Education Department (No. GJJ170759).

\section{References}

[1] W.-S. Cheung, Mappings on triangular algebras, University of Victoria, 2000.

[2] J. Vukman, "On (m,n)-Jordan centralizers in rings and algebras," Glasnik Mateematicki, vol. 45(65), no. 1, pp. 43-53, 2010.

[3] M. Bresar and B. Zalar, "On the structure of Jordan *-derivations," Colloquium Mathematicum, vol. 63, no. 2, pp. 163-171, 1992.

[4] B. Zalar, "On centralizers of semiprime rings," Commentationes Mathematicae, vol. 32, no. 4, pp. 609-614, 1991.

[5] J. Vukman, "An identity related to centralizers in semiprime rings," Commentationes Mathematicae, vol. 40, no. 3, pp. 447456, 1999.

[6] D. Benkovic and D. Eremita, "Characterizing left centralizers by their action on a polynomial," Publicationes Mathematicae, vol. 64, no. 3-4, pp. 343-351, 2004.

[7] J. Vukman and I. Kosi-Ulbl, "Centralisers on rings and algebras," Bulletin of the Australian Mathematical Society, vol. 71, no. 2, pp. 225-234, 2005.

[8] X. F. Qi, S. P. Du, and J. C. Hou, "Characterization of centralizers," Acta Mathematica Sinica, vol. 51, no. 3, pp. 509-516, 2008.

[9] C. Yang and J. H. Zhang, "Generalized Jordan centralizers on nest algebras," Acta Mathematica Sinica, vol. 53, no. 5, pp. 975980, 2010.

[10] P. Li, D. Han, and W.-S. Tang, "Centralizers and Jordan derivations for CSL subalgebras of von Neumann algebras," The Journal of Operator Theory, vol. 69, no. 1, pp. 117-133, 2013.

[11] Q. Chen, X. Fang, and C. Li, “The characterization of generalized Jordan centralizers on algebras," Sociedade Paranaense de Matemática, vol. 35, no. 3, pp. 225-240, 2017.

[12] S. H. Ali, A. Fosner, M. Fosner, and M. S. Khan, "On Generalized Jordan Triple (a, $ß)^{*}$-Derivations and Related Mappings," Mediterranean Journal of Mathematics, vol. 10, pp. 1657-1668, 2013.

[13] W.-S. Cheung, “Commuting maps of triangular algebras," Journal Of The London Mathematical Society-Second Series, vol. 63, no. 1, pp. 117-127, 2001.

[14] A. Fosner, "A note on generalized ( $m, n)$-Jordan centralizers," Demonstratio Mathematica, vol. 46, no. 2, pp. 257-262, 2013.

[15] M. E. Gordji and H. Khodaei, "A fixed point technique for investigating the stability of $(\alpha, \beta, \gamma)$-derivations on Lie $\mathrm{C}^{\star}$-algebras," Nonlinear Analysis, vol. 76, pp. 52-57, 2013. 
[16] H. Khodaei, "On the hyperstability of (m,n)-derivations," Fixed Point Theory. An International Journal on Fixed Point Theory, Computation and Applications, vol. 18, no. 2, pp. 641-650, 2017.

[17] F. Lu, “The Jordan structure of CSL algebras," Studia Mathematica, vol. 190, no. 3, pp. 283-299, 2009.

[18] Q. Shen, J. Li, and J. Guo, "On (m,n)-derivations of some algebra," Demonstratio Mathematica, vol. 47, no. 3, pp. 672-694, 2014.

[19] J. Vukman, "Centralizers on semiprime rings," Commentationes Mathematicae, vol. 42, no. 2, pp. 237-245, 2001.

[20] J. Vukman, I. Kosi-Ulbl, and D. Eremita, "On certain equations in rings," Bulletin of the Australian Mathematical Society, vol. 71, no. 1, pp. 53-60, 2005. 


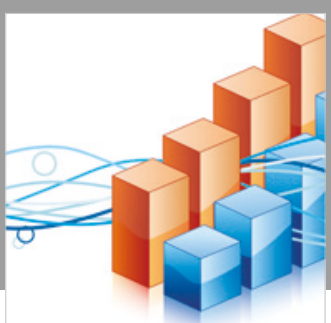

Advances in

Operations Research

\section{-n-m}
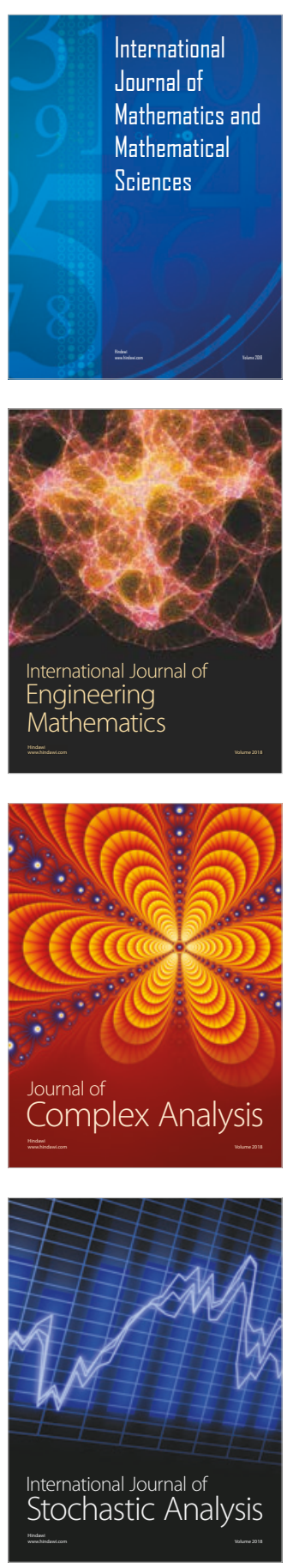
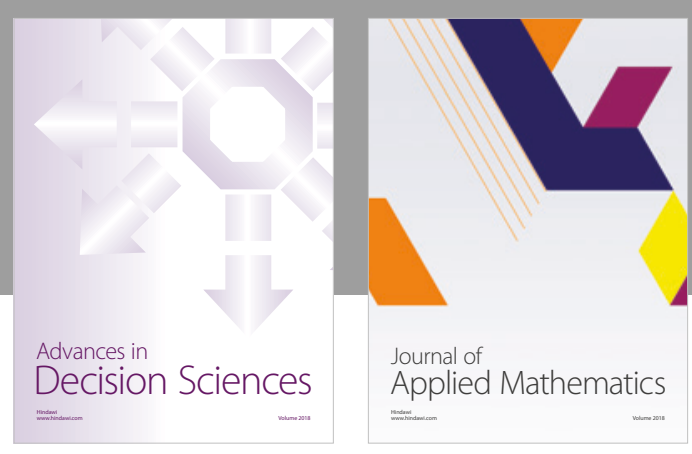

Journal of

Applied Mathematics
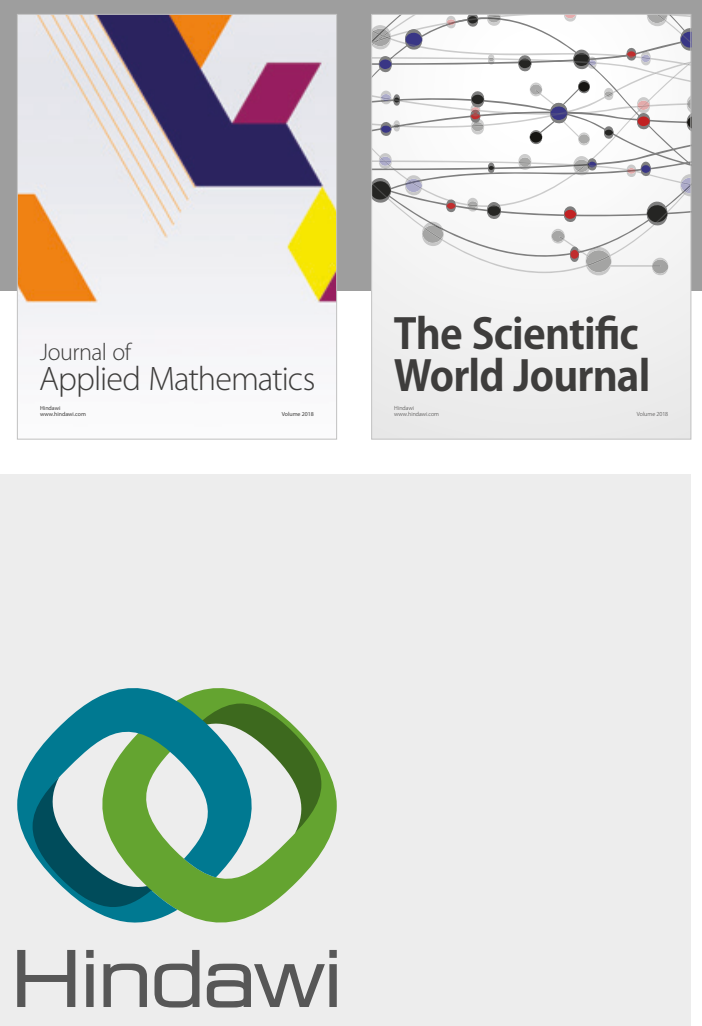

Submit your manuscripts at

www.hindawi.com

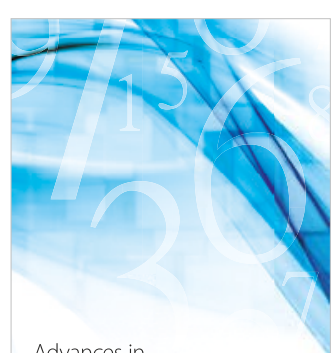

Advances in
Numerical Analysis
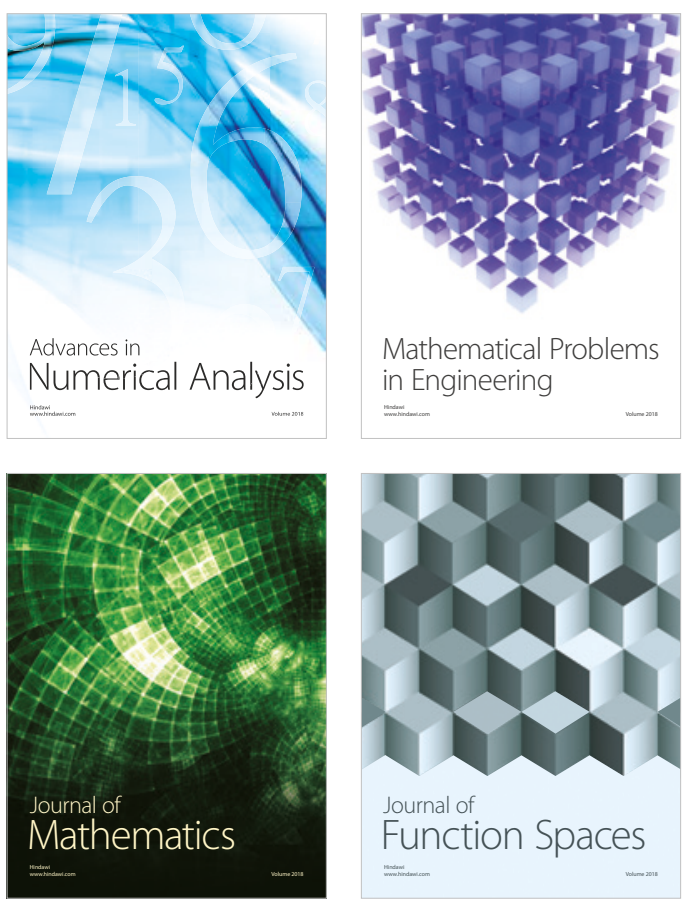

Mathematical Problems in Engineering

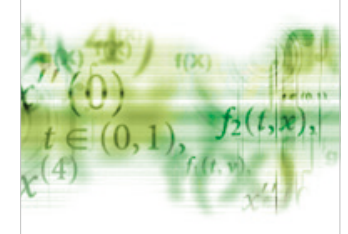

International Journal of

Differential Equations

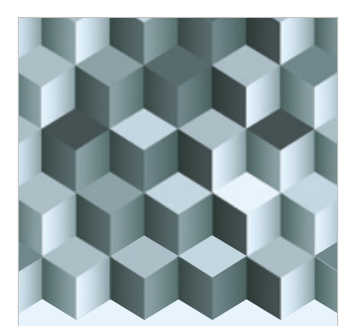

Journal of

Function Spaces

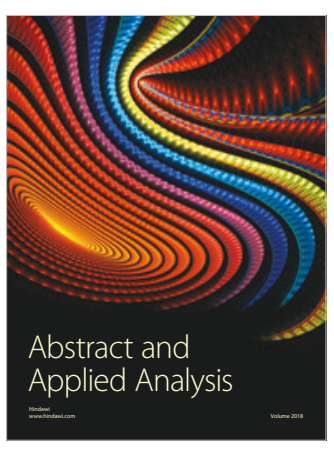

The Scientific

World Journal

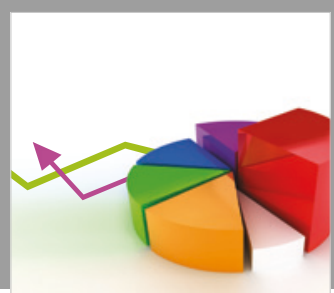

Journal of

Probability and Statistics
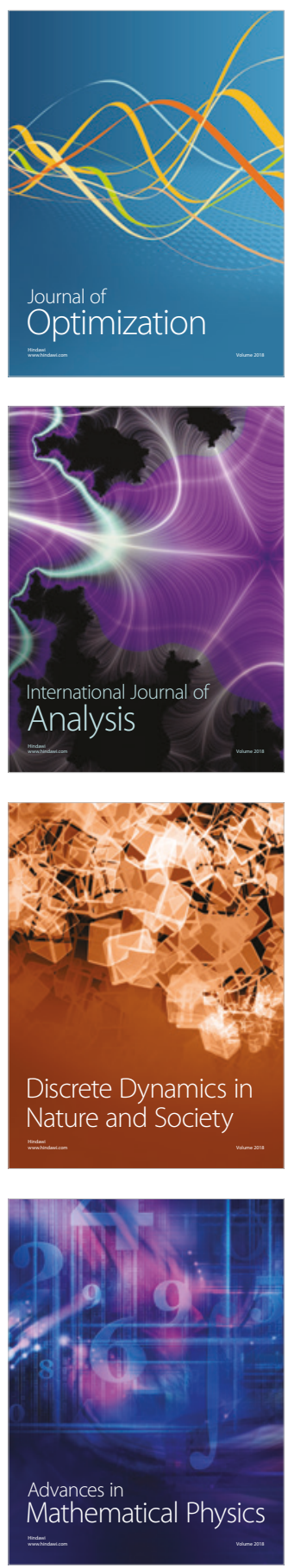